\title{
Quantum Phase Transition Induced by Magnetic Impurity
}

\section{Triangular Lattice with On-Site Pairing Study}

\author{
Szczepan Głodzik ${ }^{1}$ - Andrzej Ptok ${ }^{1,2}$ (1)
}

Received: 9 September 2017 / Accepted: 13 September 2017 / Published online: 21 September 2017

(C) The Author(s) 2017. This article is an open access publication

\begin{abstract}
The Yu-Shiba-Rusinov bound states can arise around magnetic impurities in conventional superconductors. Competition between screened and unscreened states can lead to the quantum phase transition. We discuss basic properties of this quantum phase transition in the case of the triangular lattice with on-site pairing. We show that the quantum phase transistion results from the change of the ground state of the system.
\end{abstract}

Keywords Magnetic impurity · Quantum phase transition

\section{Introduction}

Interplay between impurities and superconductors is the focus of many studies [1]. Bound states near magnetic impurities in the conventional s-wave superconductor are an example of such interplay. This behavior was proposed by Yu [2], Shiba [3] and Rusinov [4] in the 1960s. In those pioneering papers, authors describe the possibility of pair breaking by a single magnetic impurity, that leads to emergence of in-gap quasi-particles states.

Recent experiments [5-7] show the possibility of realization of this bound state, called Yu-Shiba-Rusinov (YSR)

Andrzej Ptok

aptok@mmj.pl

Szczepan Głodzik

szglodzik@kft.umcs.lublin.pl

1 Institute of Physics, Maria Curie-Skłodowska University, Plac M. Skłodowskiej-Curie 1, PL-20031 Lublin, Poland

2 Institute of Nuclear Physics, Polish Academy of Sciences, ul. E. Radzikowskiego 152, PL-31342 Kraków, Poland bound state in $\mathrm{NbSe}_{2}$ with a quasi-two dimensional (2D) triangular lattice $[8,9]$. A change in the coupling strength between the magnetic impurity and superconductor, can lead to the quantum phase transition (QPT) [10]. The main idea of this QPT is shown schematically in Fig. 1. When the coupling is weak, there are numerous bound Cooper pairs (left part). However, after QPT, when the coupling is strong enough, the local magnetic field, generated by the impurity, leads to pair breaking and formation of bound broken pairs (right panel).

In this paper, we discuss basic properties of the QPT in the case of triangular lattice by studying the evolution of YSR states using the Bogoliubov-de Gennes (BdG) equations. Our microscopic model and the BdG technique are shown in Section 2. Numerical results and discussion are presented in Section 3, and we summarize our work in Section 4.

\section{Model and Methods}

We study a 2D triangular lattice, described by attractive Hubbard model $(U<0)$. The Hamiltonian is given as:

$\mathcal{H}=H_{0}+H_{\text {imp }}+H_{s c}$.

Here

$H_{0}=-t \sum_{\langle i, j\rangle \sigma} c_{i \sigma}^{\dagger} c_{j \sigma}-\mu \sum_{i \sigma} c_{i \sigma}^{\dagger} c_{i \sigma}$

is the free-electron kinetic term. $c_{i \sigma}^{\dagger}\left(c_{i \sigma}\right)$ denotes creation (annihilation) of electron with spin $\sigma=\{\uparrow, \downarrow\}$ at $i$-th lattice site, $t$ is the hopping integral between nearest neighbors and $\mu$ is the chemical potential. 
Fig. 1 Schematic representation of the main idea of the quantum phase transition in the presence of the magnetic impurity (big red arrow) in a conventional superconductor (shown by condensate of the singlet Cooper pairs). Quantum phase transition leads to a change of the ground state from bound states of non-broken (left) to bound states of broken (right) Cooper pairs

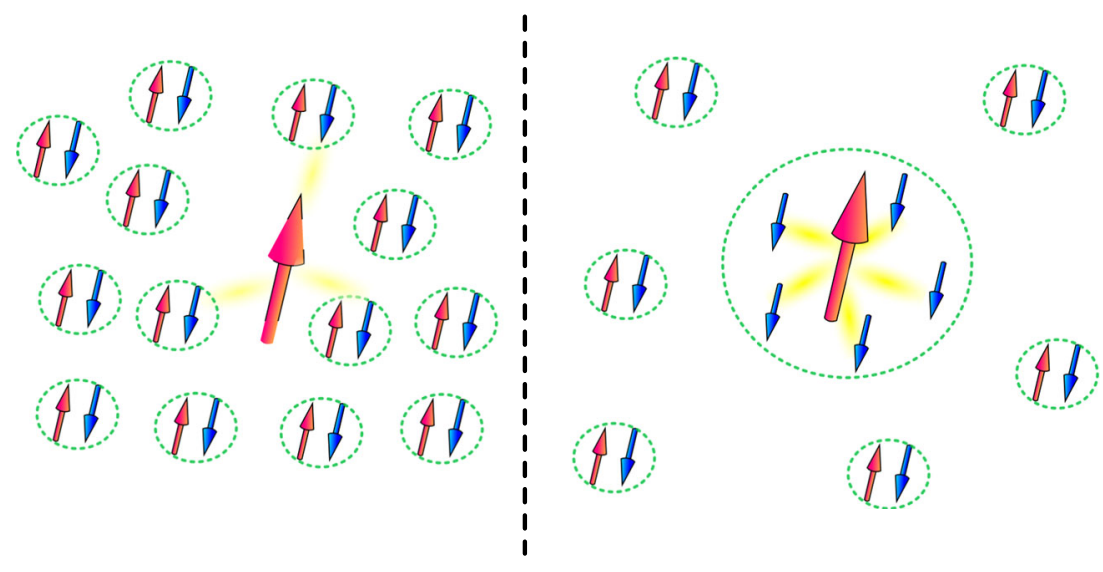

The BdG (7) can be solved self-consistently, with respect to the SOP, which can be found as

$$
\begin{aligned}
\Delta_{i} & =\left\langle c_{i \downarrow} c_{i \uparrow}\right\rangle \\
& =\sum_{n}\left(u_{i n \downarrow} v_{i n \uparrow}^{*} f\left(\mathcal{E}_{n \uparrow}\right)-u_{i n \uparrow} v_{i n \downarrow}^{*} f\left(-\mathcal{E}_{n \downarrow}\right)\right),
\end{aligned}
$$

where $f(\omega)=1 /\left(1+\exp \left(\omega / k_{B} T\right)\right)$ is the Fermi-Dirac distribution. Similar, the local density of states (LDOS) for particles with given spin $\sigma$ in $i$-th site [12] can be calculated from

$\rho_{i \sigma}(\omega)=\sum_{n}\left[\left|u_{i n \sigma}\right|^{2} \delta\left(\omega-\mathcal{E}_{n}\right)+\left|v_{i n \sigma}\right|^{2} \delta\left(\omega+\mathcal{E}_{n}\right)\right]$.

whereas (total) density of states (DOS) $\rho(\omega)=$ $\sum_{i \sigma} \rho_{i \sigma}(\omega)$.

The BdG equations method [13] can be useful in treating the general problem of superconducting state in a presence of the inhomogeneity like scalar non-magnetic impurity in a $s$-wave [14-17] or $d$-wave [17-19] superconductors, superconductivity in the presence of spin density wave [20] or vortices [21-23], ultracold Fermi gases in the external trap $[24,25]$ or unconventional FFLO superconducting phase [17, 18, 26, 27]. Moreover, this type of problems can be solved in different types of geometries, i.e., rings [28] or frames [29].

\section{Numerical Results and Discussion}

In this section, we turn our attention to numerical results for a 2D triangular lattice with $N_{x} \times N_{y}=41 \times 41$ sites in real space. In our calculations we assume $U / t=-3, \mu / t=0$ and $k_{B} T / t=0$.

In the first step, we calculate the total DOS as a function of $J$ (Fig. 2a). Constant value of $U$ leads to superconducting gap (SG) of about 0.4 in the absence of impurity $(J / t=0)$. With increasing $|J|$ we can observe the appearence of a bound state induced by magnetic impurity inside the SG 


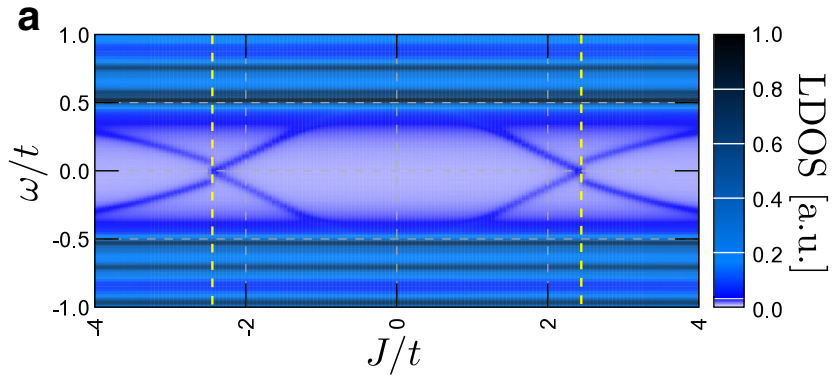

b
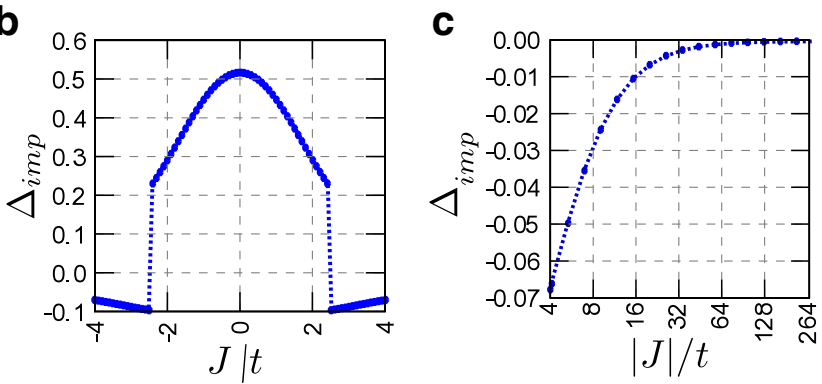

Fig. 2 DOS (a) and value of the SOP at the impurity site $\Delta_{i m p}$ (b and c) for different values of $J$. Results for $U=-3 t, \mu=0 t$ and $k_{B} T=0$

$(|\omega| / t<0.4)$. For any value of $|J|$, eigenvalues corresponding to those bound states are located symmetrically around $\omega / t=0$. Different spectral weight and opposite spin polarisation of positive and negative eigenvalues stems from the fact that YSR states are induced by magnetic impurity. In this sense, we can tell about spin polarized bound states [30], corresponding to nonzero value of $\rho_{i \uparrow}(\omega)-$ $\rho_{i \downarrow}(\omega)$.

As we can see, in some critical value of $J$ (show by yellow dashed line), the polarization of bound state are changed. This critical values $J_{c}$ correspond to QPT induced by magnetic impurity. One can observe that when $J \rightarrow J_{c}$ eigenvalues corresponding to YSR states are approaching $\omega / t=0$. Moreover, during the QPT energy of bound states changes discontinuously.

Other signature of the QPT in this system is the sign change of the SOP at the impurity site $\Delta_{i m p}$ (Fig. 2b). On other hand, like in the case of a scalar impurity, increasing $J$ leads to a decrease of $\left|\Delta_{i m p}\right|$, i.e., when $J / t \rightarrow \pm \infty$ then $\left|\Delta_{i m p}\right| \rightarrow 0$.

When describing a QPT induced by magnetic impurity, it is important to look into the total energy of the system, by calculating the grand canonical potential $\Omega(8)$. For fixed parameter, the energy is only a function of the spatial decomposition of SOP in real space, i.e., $\Omega \equiv \Omega(\boldsymbol{\Delta})$. Here, $\boldsymbol{\Delta}=\left(\Delta_{1}, \Delta_{2}, \cdots\right)$ is a $\mathrm{N}_{x} \times \mathrm{N}_{y}$-component vector of the SOP at every site of real space. To compare the $\Omega$ of a system before and after QPT, we calculate the grand canonical potential in two cases: when the SOP at the impurity site is (i) positive and (ii) negative. We denote the grand canonical

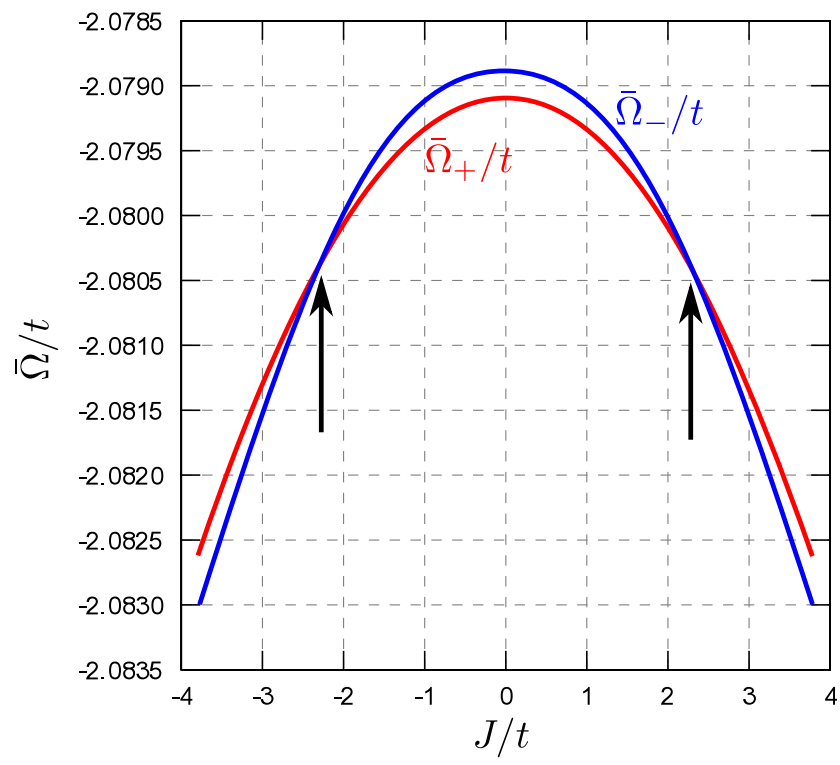

Fig. 3 Compare of the grand canonical potential per state $\bar{\Omega}=\Omega / N$ for different quantum phases, in which $\Delta_{i m p}$ are positive (red line) or negative (blue line). Results form parameter from Fig. 2

potential in these two cases $\bar{\Omega}_{+}$and $\bar{\Omega}_{-}$, respectively. The comparison of those quantities is shown in Fig. 3, where the QPTs are marked by black arrows. As we can see the minimum energy of the system is given by $\bar{\Omega}_{+}$for $|J|<\left|J_{c}\right|$, while in other cases by $\bar{\Omega}_{-}$.

The QPTs induced by magnetic impurities are similar to the classical phase transitions e.g. from superconducting to normal state. On one hand, energy of the system is given by a global minimum of the energy of different states (bound Cooper pairs or broken pair bound states). When a change of ground state is more favorable energetically, we observe a phase transition. On the other hand, QPTs are discontinuous. This can be deduced from the fact that the eigenvalues of YSR states change discontinuously and from the sign change of the SOP at the impurity site.

\section{Summary}

The magnetic impurity in a conventional superconductor can lead to a quantum phase transition. In this paper, we described this possibility in the case of triangular lattice with on-site pairing. We described basic properties of the quantum phase transition induced by the the magnetic impurity, i.e., the change of polarization of the bound states, discontinuous change of energy of bound states induced by impurity and the sign change of the superconducting order parameter at the impurity site. We have shown that the quantum phase transition is connected to a change of ground state of the system, which chooses the more energetically favorable state. 
Acknowledgements This work was supported by the National Science Centre (NCN, Poland) under grants DEC-2014/13/B/ST3/04451 (S.G.) and UMO-2016/20/S/ST3/00274 (A.P.).

Open Access This article is distributed under the terms of the Creative Commons Attribution 4.0 International License (http:// creativecommons.org/licenses/by/4.0/), which permits unrestricted use, distribution, and reproduction in any medium, provided you give appropriate credit to the original author(s) and the source, provide a link to the Creative Commons license, and indicate if changes were made.

\section{References}

1. Balatsky, A.V., Vekhter, I., Zhu, J.X.: Impurity-induced states in conventional and unconventional superconductors. Rev. Mod. Phys. 78, 373 (2006). https://doi.org/10.1103/RevModPhys.78.373

2. Yu, L.: Bound state in superconductors with paramagnetic impurities. Acta Phys. Sin. 21, 75 (1965). https://doi.org/10.7498/aps. 21.75

3. Shiba, H.: Classical spins in superconductors. Progr. Theor. Exp. Phys. 40, 435 (1968). https://doi.org/10.1143/PTP.40.435

4. Rusinov, A.I.: Sov. JETP Lett. 9, 85 (1969)

5. Ménard, G.C., Guissart, S., Brun, C., Pons, S., Stolyarov, V.S., Debontridder, F., Leclerc, M.V., Janod, E., Cario, L., Roditchev, D., Simon, P., Cren, T.: Coherent long-range magnetic bound states in a superconductor. Nat. Phys. 11, 1013 (2015). https://doi.org/10.1038/nphys3508

6. Hatter, N., Heinrich, B.W., Ruby, M., Pascual, J.I., Franke, K.J.: Magnetic anisotropy in Shiba bound states across a quantum phase transition. Nat. Commun. 6, 8988 (2015). https://doi.org/10.1038/ ncomms 9988

7. Kezilebieke, S., Dvorak, M., Ojanen, T., Liljeroth, P.: Coupled Yu-Shiba-Rusinov states in molecular dimers on $\mathrm{NbSe}_{2}$. arXiv:1701.03288 (2017)

8. Xi, X., Wang, Z., Zhao, W., Park, J.H., Law, K.T., Berger, H., Forro, L., Shan, J., Mak, K.F.: Ising pairing in superconducting $\mathrm{NbSe}_{2}$ atomic layers. Nat. Phys. 12, 139-143 (2016). https://doi.org/10.1038/nphys3538

9. Ugeda, M.M., Bradley, A.J., Zhang, Y., Onishi, S., Chen, Y., Ruan, W., Ojeda-Aristizabal, C., Ryu, H., Edmonds, M.T., Tsai, H.-Z., Riss, A., Mo, S.-K., Lee, D., Zettl, A., Hussain, Z., Shen, Z.-X., Crommie, M.F.: Characterization of collective ground states in single-layer $\mathrm{NbSe}_{2}$. Nat. Phys. 12, 92 (2016). https://doi.org/10. 1038/nphys 3527

10. Franke, K.J., Schulze, G., Pascual, J.I.: Competition of superconducting phenomena and Kondo screening at the nanoscale. Science 332, 940 (2011). https://doi.org/10.1126/science.1202204

11. De Gennes, P.G.: Superconductivity of metals and alloys. Advanced Books Classics Series. Westview Press, Boulder (1999)

12. Matsui, H., Sato, T., Takahashi, T., Wang, S.C., Yang, H.B., Ding, H., Fujii, T., Watanabe, T., Matsuda, A.: BCS-like bogoliubov quasiparticles in high- $\mathrm{T}_{c}$ superconductors observed by angleresolved photoemission spectroscopy. Phys. Rev. Lett. 90(217), 002 (2003). https://doi.org/10.1103/PhysRevLett.90.217002

13. Han, Q.: A method of studying the Bogoliubov-de Gennes equations for the superconducting vortex lattice state. J. Phys.: Condens. Matter 22(035), 702 (2010). https://doi.org/10.1088/09538984/22/3/03570

14. Maśka, M.M., Śledź, Z., Czajka, K., Mierzejewski, M.: Inhomogeneity-induced enhancement of the pairing interaction in cuprate superconductors. Phys. Rev. Lett. 99(147), 006 (2007). https://doi.org/10.1103/PhysRevLett.99.147006

15. Krzyszczak, J., Domański, T., Wysokiński, K.I., Micnas, R., Robaszkiewicz, S.: Real space inhomogeneities in high temperature superconductors: the perspective of the two-component model. J. Phys.: Condens. Matter 22(255), 702 (2010). https://doi.org/10.1088/0953-8984/22/25/255702

16. Ptok, A., Kapcia, K.J.: Probe-type of superconductivity by impurity in materials with short coherence length: the s- wave and $\eta$-wave phases study. Supercond. Sci. Technol. 28(045), 022 (2015). https://doi.org/10.1088/0953-2048/28/4/045022

17. Wang, Q., Hu, C.R., Ting, C.S.: Impurity-induced configurationtransition in the Fulde-Ferrell-Larkin-Ovchinnikov state of a $d$-wave superconductor. Phys. Rev. B 75(184), 515 (2007). https://doi.org/10.1103/PhysRevB.75.184515

18. Yanase, Y.: The disordered Fulde-Ferrel-Larkin-Ovchinnikov state in d-wave superconductors. New J. Phys. 11(055), 056 (2009). https://doi.org/10.1088/1367-2630/11/5/055056

19. Schmid, M., Loder, F., Kampf, A.P., Kopp, T.: Disorder induced stripes in d -wave superconductors. New J. Phys. 15(073), 049 (2013). https://doi.org/10.1088/1367-2630/15/7/073049

20. Ptok, A., Maśka, M.M., Mierzejewski, M.: Coexistence of superconductivity and incommensurate magnetic order. Phys. Rev. B 84(094), 526 (2011). https://doi.org/10.1103/PhysRevB.84.094526

21. Mierzejewski, M., Maśka, M.M.: Critical field in a superconductivity model with local pairs. Phys. Rev. B 69(054), 502 (2004). https://doi.org/10.1103/PhysRevB.69.054502

22. Kim, W., Chen, Y., Ting, C.S.: Impurity resonance peaks in the vortex core of cuprate superconductors with induced spin density wave order. Phys. Rev. B 80(172), 502 (2009). https://doi.org/10.1103/PhysRevB.80.172502

23. Nagai, Y., Nakai, N., Machida, M.: Direct numerical demonstration of sign-preserving quasiparticle interference via an impurity inside a vortex core in an unconventional superconductor. Phys. Rev. B 85(092), 505 (2012). https://doi.org/10.1103/PhysRevB. 85.092505

24. Kinnunen, J., Jensen, L.M., Törmä, P.: Strongly interacting fermi gases with density imbalance. Phys. Rev. Lett. 96(110), 403 (2006). https://doi.org/10.1103/PhysRevLett.96.110403

25. Baksmaty, L.O., Lu, H., Bolech, C.J., Pu, H.: A Bogoliubov-de Gennes study of trapped spin-imbalanced unitary Fermi gases. New J. Phys. 13(055), 014 (2011). https://doi.org/10.1088/13672630/13/5/055014

26. Wang, Q., Chen, H.Y., Hu, C.R., Ting, C.S.: Local tunneling spectroscopy as a signature of the Fulde-Ferrell-Larkin-Ovchinnikov state in $s$ - and $d$-wave superconductors. Phys. Rev. Lett. 96(117), 006 (2006). https://doi.org/10.1103/PhysRevLett.96.117006

27. Ptok, A.: The Fulde-Ferrell-Larkin-Ovchinnikov superconductivity in disordered systems. Acta Phys. Pol. A 118, 420 (2010). https://doi.org/10.12693/APhysPolA.118.420

28. Ptok, A.: The Fulde-Ferrell-Larkin-Ovchinnikov state in quantum rings. J. Supercond. Nov. Magn. 25, 1843 (2012). https://doi. org/10.1007/s10948-012-1574-5

29. Zha, G.Q., Covaci, L., Peeters, F.M., Zhou, S.P.: Majorana fermion states and fractional flux periodicity in mesoscopic $d$ wave superconducting loops with spin-orbit interaction. Phys. Rev. B 90(014), 522 (2014). https://doi.org/10.1103/PhysRevB. 90.014522

30. Yazdani, A., Jones, B.A., Lutz, C.P., Crommie, M.F., Eigler, D.M.: Probing the local effects of magnetic impurities on superconductivity. Science 275, 1767 (1997). https://doi.org/10.1126/science. 275.5307.1767 